\title{
Not doomed to fail
}

\section{Anne Taormina ${ }^{a}$ and Katrin Wendland ${ }^{b}$}

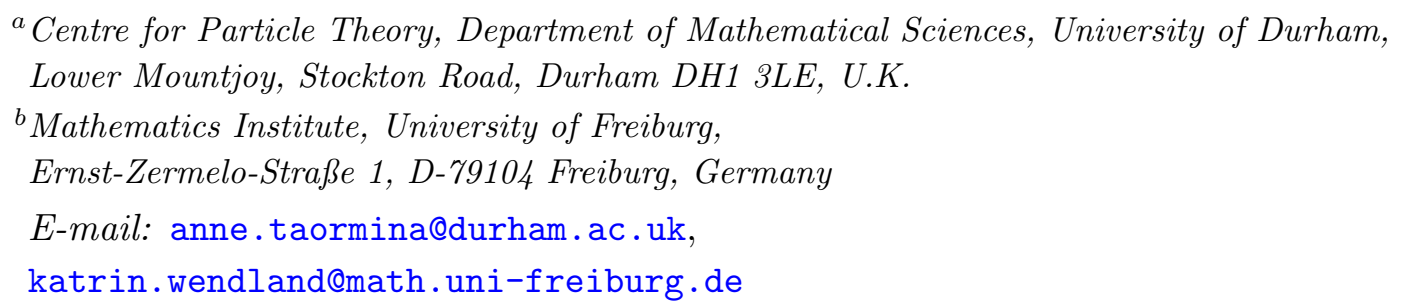

AbStRaCT: In their recent manuscript "An uplifting discussion of T-duality" [26], J. Harvey and G. Moore have reevaluated a mod two condition appearing in asymmetric orbifold constructions as an obstruction to the description of certain symmetries of toroidal conformal field theories by means of automorphisms of the underlying charge lattice. The relevant "doomed to fail" condition determines whether or not such a lattice automorphism $g$ may lift to a symmetry in the corresponding toroidal conformal field theory without introducing extra phases. If doomed to fail, then in some cases, the lift of $g$ must have double the order of $g$. It is an interesting question, whether or not "geometric" symmetries are affected by these findings. In the present note, we answer this question in the negative, by means of elementary linear algebra: "geometric" symmetries of toroidal conformal field theories are not doomed to fail. Consequently, and in particular, the symmetry groups involved in symmetry surfing the moduli space of K3 theories do not differ from their lifts.

KEYwords: Conformal Field Theory, Discrete Symmetries, Sigma Models

ARXiv EPrint: 1708.01563 


\section{Contents}

1 Geometric symmetries of K3 theories

2 Not doomed to fail

Introduction. Symmetries are a driving force in many areas of mathematics and theoretical physics. They are, in particular, central in the investigation of the recent phenomenon of Mathieu Moonshine and its relations to K3 theories [1-11]. The Torelli theorems for complex tori [12] and K3 surfaces [13-17] allow a description of the symmetries of these complex surfaces in terms of automorphisms of the respective lattices of integral cohomology. Inspired by these theorems, the use of lattice automorphisms has been extended to the investigation and classification of symmetries of superconformal field theories whose targets are complex surfaces $[18,19]$.

At the basis of this approach lies, roughly speaking, the description of the moduli spaces of K3 theories [20-23], on the one hand, and of toroidal conformal field theories [24, 25], on the other, as Grassmannians that are modelled on the even or odd part of the total cohomology of the complex two-dimensional target manifold. This may be viewed as an extension of the description of the moduli spaces of hyperkähler structures for the respective complex surfaces in terms of Grassmannians, which are modelled on the second cohomology. It is thus natural to expect the properties of automorphisms of the underlying lattices of integral cohomology to allow for a generalization to the conformal field theory setting. Though well rooted in mathematics, the very description of general symmetries both of toroidal and of $\mathrm{K} 3$ theories in terms of such lattice automorphisms is not immediate. The more important are the recent results [26] by J. Harvey and G. Moore, which show that the symmetries of toroidal conformal field theories cannot, in general, be fully described in terms of their action on the underlying charge lattice. Applied to toroidal $N=(4,4)$ superconformal field theories at central charges $c=\bar{c}=6$, where the charge lattice may be directly related to the lattice of odd integral cohomology of the target [23, 27], this raises some interesting questions about the traditional discussions of symmetries for these models.

More precisely, this discussion concerns symmetries of a toroidal conformal field theory which induce an action on the underlying charge lattice fixing the respective parameter point in the Grassmannian description of the moduli space. In [26], it is shown that vice versa, for certain toroidal conformal field theories, there exist automorphisms of the charge lattice which preserve the parameter point in the moduli space, but which can only lift to symmetries of at least double the order for the corresponding conformal field theory. In other words, the charge lattice of such a theory does not fully capture the symmetry group of the conformal field theory. There are also cases where a lift of the same order exists, which however acts non-trivially on some winding-momentum fields associated to invariant charge 
vectors. ${ }^{1}$ Both phenomena could potentially cast some doubt on traditional descriptions of symmetries in conformal field theory. Whether or not either of these phenomena occurs for a given lattice automorphism is encoded by equation (2.17) of the manuscript [26], simply dubbed the "doomed to fail" condition. This condition had previously been stated with different interpretation by K.S. Narain, M.H. Sarmadi and C. Vafa [28, 29], and in a slightly different context by J. Lepowsky [30, 31]. Hai Siong Tan used a similar approach in [32] to determine consistent asymmetric orbifold group actions. The condition can be traced back to the properties of involutions on the charge lattice which fix the parameter point: whenever there exists a charge vector which has an odd scalar product with its image under such an involution, the lift is "doomed to fail", i.e. either only a lift of order four exists, or there are invariant charge vectors whose associated momentum-winding fields are multiplied by $(-1)$. The latter phenomenon of "non-trivial phases" is compatible with the description of symmetries of toroidal sigma models [19], and it had been taken into account in previous discussions of symmetries in conformal field theory [18, 19, 33]. The former phenomenon, which affects the relevant groups of symmetries, has not been discussed in this form before. The criterion for at least one of these two phenomena to occur is very elegant, as it makes it a simple matter of linear algebra to check whether or not a given lattice automorphism is doomed.

These findings could well be of direct relevance for the discussion of Mathieu Moonshine, independently of the general doubts that they may cast on the use of lattice techniques in describing symmetries of conformal field theories. Indeed, by a $\mathbb{Z}_{2}$-orbifolding, any toroidal $N=(4,4)$ superconformal field theory at central charges $c=\bar{c}=6$ gives rise to a $\mathrm{K} 3$ theory. The $\mathbb{Z}_{2}$-orbifolding procedure thereby induces a map between the respective moduli spaces which was determined in [23]. This map is described in terms of the underlying lattices of integral cohomology, and it requires a transition between the odd and the even integral cohomology of complex two-tori by means of triality [23]. ${ }^{2}$ The symmetries of the underlying toroidal conformal field theories thus induce symmetries of the resulting K3 theories. A failure of a lattice automorphism to fully capture a symmetry of a toroidal theory may descend to the corresponding K3 theory.

We are not able to predict the full scope of consequences for Mathieu Moonshine that may follow from [26]. However, in this note we come back to a remark made by J. Harvey and G. Moore in the first version of their recent paper, addressing symmetry surfing. The latter is a technique that we have proposed first in [34] and that allows to combine geometric symmetry groups from distinct points in the moduli space of K3 theories [6]. In [9], we have shown that the maximal subgroup $\mathbb{Z}_{2}^{4}: A_{8}$ of $M_{24}$ is the group which, by means of symmetry surfing, combines all geometric symmetries induced by the symmetries of complex tori in the corresponding $\mathbb{Z}_{2}$-orbifold conformal field theories. By constructing the leading order massive representation which contributes to Mathieu Moonshine, for this maximal subgroup, our work [8] provides the first piece of evidence that the relevant

\footnotetext{
${ }^{1}$ We thank G. Moore for emphasizing this point to us.

${ }^{2}$ Note that at this point, one needs to work on a $2: 1$ cover of the moduli space of toroidal superconformal field theories in order to keep the target space orientation, as is required for the resulting K3 theories [23, eq. (1.17)].
} 
representations of Mathieu Moonshine might arise intrinsically from conformal field theory. Our arguments have, in the meantime, been vastly generalized by M. Gaberdiel, Ch. Keller and H. Paul [35], yielding additional evidence in favour of the proposal of symmetry surfing.

Whether or not the symmetries that are relevant for the works $[6,8,9,35]$ are doomed to fail is an important question for our programme. In this note we show that thanks to the very elegant "doomed to fail" condition, this question may be answered in the negative by means of elementary linear algebra: symmetry surfing is not doomed to fail by the uplifting properties of lattice automorphisms. It is important to appreciate that this statement is closely tied to the fact that symmetry surfing only proposes to combine geometric symmetries of K3 theories. In fact, we show more generally that geometric symmetries of toroidal conformal field theories are never doomed to fail. The very definition of geometric symmetries needs to be treated with great care. It refers to symplectic automorphisms of finite order that also leave invariant the B-field, viewed as a real-valued two-form. In particular, this notion excludes the identification of a B-field with its shifts by integral cohomology classes, thus excluding some symmetries that are certainly in the realm of geometry. ${ }^{3}$ The adjective geometric thus solely means that such symmetries leave invariant a geometric interpretation. In the context of symmetry surfing, our notion of geometric symmetries is inseparably connected with the idea that there is a space of states that generically exist in all K3 theories, which bears all the structure that is relevant to Mathieu Moonshine. As already predicted in $[9,36]$, the cohomology of the chiral de Rham complex of [37$42]$ is expected to model this "space of generic states". In identifying the cohomology of the chiral de Rham complex with the large volume limit of a topological half-twist of K3 theories [43], we need to require compatibility of the symmetries in question with a large volume limit. We do so by requiring that they leave invariant a geometric interpretation of the model. Recently, the idea of explaining Mathieu Moonshine by means of such a space of generic states has been further substantiated by the observation that the cohomology of the chiral de Rham complex for K3 surfaces indeed decomposes into irreducible representations of the "small" $N=4$ superconformal algebra at central charge $c=6$ with proper multiplicity spaces of every massive representation, i.e. without the occurrence of virtual representations $[10,11]$.

We emphasize that symmetry surfing has not been proved to explain Mathieu Moonshine, so far, and that this proposal may still ultimately fail, as is extensively discussed in [11, section 4.5]. However, in this note we use plain scientific arguments to prove that the symmetry surfing programme is not jeopardized by misidentification of geometric symmetries at the level of toroidal conformal field theories. Nevertheless, we expect that the findings of [26] have important implications on the discussion of symmetries of K3 theories, and thus on the broader programme.

The remainder of this note is divided into two sections. Section 1 is devoted to a more detailed discussion of our notion of geometric symmetries. We describe the properties of the symmetries that enter the symmetry surfing proposal of $[6,9,34]$ and that are thus

\footnotetext{
${ }^{3} \mathrm{~A}$ lower-dimensional toroidal example is a reflection in a simple root in the $\mathfrak{s u}(3)$-point for two free bosons, discussed in [26, section 4.1]. We thank G. Moore for this comment.
} 
relevant for $[8,35]$. In particular, the properties of the underlying symmetries of toroidal conformal field theories and their induced actions on the respective charge lattices are discussed. Section 2 is devoted to the proof of our claim that geometric symmetries of toroidal conformal field theories, in particular those that enter symmetry surfing, are not doomed to fail. We remark that an alternative, just as immediate proof follows from the discussion around equation (4.54) of [32].

\section{Geometric symmetries of K3 theories}

The Mathieu Moonshine phenomenon, discovered by T. Eguchi, H. Ooguri and Y. Tachikawa [1], predicts the existence of a $\mathbb{Z}_{2} \times \mathbb{Z}_{2}$ graded representation of the "small" $N=4$ superconformal algebra of [44] at central charge $c=6$, whose diagonally $\mathbb{Z}_{2}$-graded character yields the complex elliptic genus of a K3 surface, and which simultaneously furnishes a representation of the Mathieu group $M_{24}$. This should yield the corresponding twisted twining genera with their strongly restrictive modular properties. The existence of such an $M_{24}$-module was proved by T. Gannon [7]. However, in addition one expects a compatible structure of a super vertex operator algebra on this $M_{24}$-module. The latter has not been constructed, so far. Neither has a satisfactory explanation been found for the existence of an $M_{24}$-module with all this structure.

Our quest for an explanation of these phenomena has led us to propose that the $M_{24^{-}}$ module in question should arise as a subspace of the spaces of states of K3 theories which is common to all such theories. The representations constructed in $[8,35]$ arise precisely as such from the spaces of states of $\mathbb{Z}_{2}$-orbifold conformal field theories on K3. We suggest that the action of $M_{24}$ might then be explained by means of certain symmetry groups of $\mathrm{K} 3$ theories on this space of generic states, combined from distinct points of the moduli space. The latter is our proposal of symmetry surfing.

As explained in $[9,11,36]$, we expect such a space of generic states to arise as the large volume limit of a topological half-twist of the space of states of our K3 theories (denoted $\mathcal{X}$ in [9, section 5], for example). According to A. Kapustin, it thus may be modelled by the cohomology of the chiral de Rham complex of the underlying K3 surface [43], which according to [38, proposition 3.7 and definition 4.1] indeed carries the structure of a super vertex operator algebra, see also [10]. To be compatible with such a large volume limit, the symmetries that are relevant to Mathieu Moonshine must be geometric in the sense that they are induced by geometric symmetries of the underlying K3 surface, independently of the volume. More precisely, we require these symmetries to fix a geometric interpretation of our theories according to [22].

To characterize such symmetries more specifically, let us recall the description of the moduli space of K3 theories, following [22, 23]. Indeed, a K3 theory may be specified by data that determine a hyperkähler structure on a K3 surface $X$, its volume $V \in \mathbb{R}, V>0$, and its $B$-field $B \in H^{2}(X, \mathbb{R})$. Here, the cohomology $H^{*}(X, \mathbb{R})$ of $\mathrm{K} 3$ is equipped with the scalar product $\langle\cdot, \cdot\rangle$ of signature $(4,20)$ that is induced by the intersection form. A hyperkähler structure on $X$ may then be uniquely specified by an oriented, positive definite three-dimensional subspace $\Sigma \subset H^{2}(X, \mathbb{R})$. Denoting by $v^{0} \in H^{0}(X, \mathbb{Z}), v \in H^{4}(X, \mathbb{Z})$ a 
generating pair of vectors for the hyperbolic lattice $H^{0}(X, \mathbb{Z}) \oplus H^{4}(X, \mathbb{Z})$ with $\left\langle v^{0}, v\right\rangle=$ 1 , the K3 theory in question is uniquely specified by the positive definite oriented fourdimensional subspace of $H^{*}(X, \mathbb{R})$ which is generated by

$$
\{\sigma-\langle B, \sigma\rangle v \mid \sigma \in \Sigma\} \cup\left\{v^{0}+B+\left(V-\frac{\langle B, B\rangle}{2}\right) v\right\}
$$

As is explained, for example, in [18], the symmetries in question in particular induce lattice automorphisms of $H^{*}(X, \mathbb{Z})$ which leave the above four-dimensional subspace of $H^{*}(X, \mathbb{R})$ invariant, point-wise. To be compatible with a large volume limit, this property must hold independently of the value of $V$. It follows that the vector $v$ must be invariant under our lattice automorphisms. Since our large volume limit is not only independent of the value of $V$ but solely depends on a choice of complex structure, in all our works we have been even more restrictive on the symmetries that enter symmetry surfing. To call a symmetry geometric, we require it to fix the geometric interpretation, i.e. we require that the induced lattice automorphism fixes both $v$ and $v^{0}$. Thus $B$ must also be fixed, see [9, footnotes 18, 19], [8, section 4], and [33, section 4.1.1].

All the symmetries that have been used in symmetry surfing, so far $[6,8,9,35]$, are induced from symmetries of Kummer surfaces that in turn descend from symmetries of the underlying complex torus. In other words, any such symmetry is given in terms of the geometric interpretation of our toroidal theory on some torus $\mathbb{R}^{d} / \Lambda, d=4$, with B-field $\widetilde{B}$. Here, $\widetilde{B}$ is given by a real, skew-symmetric $d \times d$ matrix, $\Lambda \subset \mathbb{R}^{d}$ is a lattice of rank $d$ and by $\Lambda^{*} \subset \mathbb{R}^{d}$ we denote its dual after identification of $\mathbb{R}^{d}$ with $\left(\mathbb{R}^{d}\right)^{*}$ by means of the Euclidean metric $\cdot$, that is,

$$
\Lambda^{*}=\left\{\mu \in \mathbb{R}^{d} \mid \mu \cdot \lambda \in \mathbb{Z} \forall \lambda \in \Lambda\right\} .
$$

The corresponding charge lattice then is

$$
\Gamma(\Lambda, \widetilde{B})=\left\{\frac{1}{\sqrt{2}}(\mu-\widetilde{B} \lambda+\lambda ; \mu-\widetilde{B} \lambda-\lambda) \mid(\mu, \lambda) \in \Lambda^{*} \oplus \Lambda\right\} \subset \mathbb{R}^{d, d},
$$

where we use the standard conventions as for example in [23, eq. (1.11)], [33, eqs. (A.3)(A.5)], [26, eq. (3.3)], and $\mathbb{R}^{d, d}=\mathbb{R}^{d} \oplus \mathbb{R}^{d}$ is equipped with the scalar product

$$
\forall\left(p_{l} ; p_{r}\right),\left(p_{l}^{\prime} ; p_{r}^{\prime}\right) \in \Gamma(\Lambda, \widetilde{B}): \quad\left(p_{l} ; p_{r}\right) \bullet\left(p_{l}^{\prime} ; p_{r}^{\prime}\right)=p_{l} \cdot p_{l}^{\prime}-p_{r} \cdot p_{r}^{\prime}
$$

of signature $(d, d)$. The geometric symmetries of the toroidal superconformal field theory that might potentially be affected by the findings of [26] are thus given by linear maps $g \in O(d)$ with $g \Lambda=\Lambda$ and $g \widetilde{B}=\widetilde{B} g$. The induced action of $g$ on the charge lattice is

$$
\forall p=\left(p_{l} ; p_{r}\right) \in \Gamma(\Lambda, \widetilde{B}): \quad g(p):=\left(g p_{l} ; g p_{r}\right) .
$$

For concrete examples relevant to symmetry surfing, the reader is referred to [9, section 1]. We emphasize that the respective symmetry groups may be non-abelian, and that this does not impose any additional difficulties. 


\section{Not doomed to fail}

Consider a toroidal conformal field theory with charge lattice $\Gamma \subset \mathbb{R}^{d, d}$, and a lattice automorphism $\gamma$ of $\Gamma$ which fixes the parameter point of the theory, i.e. which acts on the charge lattice by means of

$$
\forall p=\left(p_{l} ; p_{r}\right) \in \Gamma: \quad \gamma(p)=\left(g_{l} p_{l} ; g_{r} p_{r}\right)
$$

with $g_{l}, g_{r} \in O(d)$. Assume that $\gamma$ has order $\ell$. Then, reevaluating obstructions previously discussed in different interpretations or contexts [28-32], J. Harvey and G. Moore find the following obstruction for $\gamma$ to lift to an automorphism of order $\ell$ of the corresponding toroidal conformal field theory that leaves invariant winding-momentum fields associated to $\gamma$-invariant charge vectors [26, eq. (2.17)]: such a lift is doomed to fail if $\ell$ is even and

$$
\exists p \in \Gamma: \quad p \bullet \gamma^{\ell / 2}(p) \notin 2 \mathbb{Z} .
$$

To show that the geometric symmetries of toroidal conformal field theories are not doomed to fail, we may thus assume without loss of generality that $\ell=2$. As explained in section 1 above, by (1.2) we furthermore assume that $g_{l}=g_{r}=g \in O(d), \Gamma=\Gamma(\Lambda, \widetilde{B})$ as in (1.1) with $\widetilde{B}^{T}=-\widetilde{B}$, and that $g \Lambda=\Lambda, g \widetilde{B}=\widetilde{B} g$. We thus have $g=g^{-1}=g^{T}$, and we find

$$
(g \widetilde{B})^{T}=-\widetilde{B} g=-g \widetilde{B} .
$$

In particular, we have

$$
\forall \lambda, \lambda^{\prime} \in \mathbb{R}^{d}: \quad \lambda \cdot(g \widetilde{B}) \lambda^{\prime}+\lambda^{\prime} \cdot(g \widetilde{B}) \lambda=0 .
$$

For charge vectors

$$
p=\frac{1}{\sqrt{2}}(\mu-\widetilde{B} \lambda+\lambda, \mu-\widetilde{B} \lambda-\lambda), \quad p^{\prime}=\frac{1}{\sqrt{2}}\left(\mu^{\prime}-\widetilde{B} \lambda^{\prime}+\lambda^{\prime}, \mu^{\prime}-\widetilde{B} \lambda^{\prime}-\lambda^{\prime}\right)
$$

with arbitrary $\lambda, \lambda^{\prime} \in \Lambda$ and $\mu, \mu^{\prime} \in \Lambda^{*}$ we thus have

$$
\begin{aligned}
p^{\prime} \bullet \gamma(p) & =\left(\mu^{\prime}-\widetilde{B} \lambda^{\prime}\right) \cdot g \lambda+\lambda^{\prime} \cdot g(\mu-\widetilde{B} \lambda) \\
& \stackrel{g=g^{T}}{=} \mu^{\prime} \cdot g \lambda+\mu \cdot g \lambda^{\prime}-\lambda \cdot(g \widetilde{B}) \lambda^{\prime}-\lambda^{\prime} \cdot(g \widetilde{B}) \lambda \\
& \stackrel{(2.2)}{=} \mu^{\prime} \cdot g \lambda+\mu \cdot g \lambda^{\prime} .
\end{aligned}
$$

In particular,

$$
\forall p=\frac{1}{\sqrt{2}}(\mu-\widetilde{B} \lambda+\lambda, \mu-\widetilde{B} \lambda-\lambda) \in \Gamma(\Lambda, \widetilde{B}): \quad p \bullet \gamma(p)=2 \mu \cdot g \lambda \in 2 \mathbb{Z}
$$

since by assumption, $g \lambda \in \Lambda$ and $\mu \in \Lambda^{*}$. In other words, the "doomed to fail" condition (2.1) does not hold. In particular, at $d=4$ we learn that the symmetries that have been relevant for symmetry surfing, so far, are not doomed to fail.

We remark that the "doomed to fail condition" of [26] is solely testing the cyclic subgroups of a given symmetry group. However, as noted at the end of section 1, symmetrysurfing does involve non-cyclic, in fact even non-abelian symmetry groups. Actually, all 
examples of symmetries that are doomed to fail and that are discussed in [26] arise in nonabelian global symmetry groups of special conformal field theories with enhanced symmetry. This raises the question of whether every geometric symmetry group $G$ has a lift to a symmetry group of the respective conformal field theory which is isomorphic to $G$. That this is indeed the case follows from the existence of an invariant 2-cocycle

$$
\varepsilon: \Gamma \times \Gamma \longrightarrow\{ \pm 1\}
$$

on the charge lattice $\Gamma$ which governs the operator product expansions between vertex operators (see, for example, [45-48] for the classical results). Indeed, with notations as in (2.3), one may use the cocycle

$$
\forall p, p^{\prime} \in \Gamma: \quad \varepsilon\left(p, p^{\prime}\right):=(-1)^{\mu \cdot \lambda^{\prime}} .
$$

For a geometric symmetry $\gamma$ as above, $g \in O(d)$ thus implies

$$
\forall p, p^{\prime} \in \Gamma: \quad \varepsilon\left(\gamma(p), \gamma\left(p^{\prime}\right)\right)=\varepsilon\left(p, p^{\prime}\right) .
$$

In terms of [26, appendix A], this means that a lift $G \longrightarrow \widehat{G}, g \mapsto T_{g}$, of our symmetry group $G$ exists which obeys $T_{g_{1}} \circ T_{g_{2}}=T_{g_{1} g_{2}}$ for all $g_{1}, g_{2} \in G$ (see [26, eqs. (A.5)-(A.11)]), thus yielding $G \cong \widehat{G}$.

\section{Acknowledgments}

We thank J. Harvey and G. Moore for communicating a preliminary version of their work [26] to us very early on, and for very useful discussions. This gave us the chance to set the record straight quickly, regarding symmetry surfing not being doomed to fail. We also thank an anonymous referee for carefully reading the manuscript and for raising a number of questions whose answers will certainly help the readers to put our work in the right context.

Open Access. This article is distributed under the terms of the Creative Commons Attribution License (CC-BY 4.0), which permits any use, distribution and reproduction in any medium, provided the original author(s) and source are credited.

\section{References}

[1] T. Eguchi, H. Ooguri and Y. Tachikawa, Notes on the K3 surface and the Mathieu group $M_{24}$, Exper. Math. 20 (2011) 91 [arXiv:1004.0956] [INSPIRE].

[2] M.C.N. Cheng, K3 surfaces, $N=4$ dyons and the Mathieu group $M_{24}$, Commun. Num. Theor. Phys. 4 (2010) 623 [arXiv:1005.5415] [INSPIRE].

[3] M.R. Gaberdiel, S. Hohenegger and R. Volpato, Mathieu twining characters for K3, JHEP 09 (2010) 058 [arXiv: 1006.0221] [INSPIRE].

[4] M.R. Gaberdiel, S. Hohenegger and R. Volpato, Mathieu moonshine in the elliptic genus of K3, JHEP 10 (2010) 062 [arXiv: 1008.3778] [INSPIRE]. 
[5] T. Eguchi and K. Hikami, Note on twisted elliptic genus of K3 surface, Phys. Lett. B 694 (2011) 446 [arXiv: 1008.4924] [INSPIRE].

[6] A. Taormina and K. Wendland, The overarching finite symmetry group of Kummer surfaces in the Mathieu group $M_{24}$, JHEP 08 (2013) 125 [arXiv:1107.3834] [INSPIRE].

[7] T. Gannon, Much ado about Mathieu, Adv. Math. 301 (2016) 322 [arXiv:1211.5531] [INSPIRE].

[8] A. Taormina and K. Wendland, A twist in the $M_{24}$ moonshine story, Confluentes Math. 7 (2015) 83 [arXiv: 1303.3221] [INSPIRE].

[9] A. Taormina and K. Wendland, Symmetry-surfing the moduli space of Kummer K3s, Proc. Symp. Pure Math. 90 (2015) 129 [arXiv:1303.2931] [INSPIRE].

[10] B. Song, Chiral Hodge cohomology and Mathieu moonshine, arXiv:1705.04060.

[11] K. Wendland, Hodge-elliptic genera and how they govern K3 theories, arXiv:1705.09904 [INSPIRE].

[12] E. Looijenga and C. Peters, Torelli theorems for K3-surfaces, Compos. Math. 42 (1981) 145.

[13] V. Kulikov, Surjectivity of the period mapping for K3 surfaces, Usp. Mat. Nauk 32 (1977) 257.

[14] E. Looijenga, A Torelli theorem for Kähler-Einstein K3 surfaces, Lect. Notes Math. 894 (1981) 107.

[15] Y. Namikawa, Surjectivity of period map for K3 surfaces, Progr. Math. 39 (1983) 379.

[16] Y. Siu, A simple proof of the surjectivity of the period map of K3 surfaces, Manuscr. Math. 35 (1981) 311.

[17] A. Todorov, Applications of the Kähler-Einstein-Calabi-Yau metric to moduli of K3 surfaces, Invent. Math. 61 (1980) 251.

[18] M.R. Gaberdiel, S. Hohenegger and R. Volpato, Symmetries of K3 sigma models, Commun. Num. Theor. Phys. 6 (2012) 1 [arXiv:1106.4315] [inSPIRE].

[19] R. Volpato, On symmetries of $\mathcal{N}=(4,4)$ sigma models on $T^{4}$, JHEP 08 (2014) 094 [arXiv: 1403.2410] [INSPIRE].

[20] N. Seiberg, Observations on the moduli space of superconformal field theories, Nucl. Phys. B 303 (1988) 286 [INSPIRE].

[21] S. Cecotti, $N=2$ Landau-Ginzburg vs. Calabi-Yau sigma models: non-perturbative aspects, Int. J. Mod. Phys. A 6 (1991) 1749 [inSPIRE].

[22] P.S. Aspinwall and D.R. Morrison, String theory on K3 surfaces, in Mirror symmetry II, B. Greene and S. Yau eds., American Mathematical Society, U.S.A. (1994), pp. 703-716 [hep-th/9404151] [INSPIRE].

[23] W. Nahm and K. Wendland, A hiker's guide to K3. Aspects of $N=(4,4)$ superconformal field theory with central charge $c=6$, Commun. Math. Phys. 216 (2001) 85 [hep-th/9912067] [INSPIRE].

[24] A. Casher, F. Englert, H. Nicolai and A. Taormina, Consistent superstrings as solutions of the $D=26$ bosonic string theory, Phys. Lett. 162B (1985) 121 [INSPIRE].

[25] K. Narain, New heterotic string theories in uncompactified dimensions $<10$, Phys. Lett. B 169 (1986) 41. 
[26] J.A. Harvey and G.W. Moore, An uplifting discussion of T-duality, JHEP 05 (2018) 145 [arXiv: 1707.08888] [INSPIRE].

[27] R. Dijkgraaf, Instanton strings and hyper-Kähler geometry, Nucl. Phys. B 543 (1999) 545 [hep-th/9810210] [INSPIRE].

[28] K.S. Narain, M.H. Sarmadi and C. Vafa, Asymmetric orbifolds, Nucl. Phys. B 288 (1987) 551 [INSPIRE].

[29] K.S. Narain, M.H. Sarmadi and C. Vafa, Asymmetric orbifolds: Path integral and operator formulations, Nucl. Phys. B 356 (1991) 163 [inSPIRE].

[30] J. Lepowsky, Calculus of twisted vertex operators, Proc. Nat. Acad. Sci. U.S.A. 82 (1985) 8295.

[31] K. Barron, Y.-Z. Huang and J. Lepowsky, An equivalence of two constructions of permutation-twisted modules for lattice vertex operator algebras, Pure and Appl. Math. 210 (2007) 797 [math/0609656] [INSPIRE].

[32] H.S. Tan, T-duality twists and asymmetric orbifolds, JHEP 11 (2015) 141 [arXiv: 1508.04807] [INSPIRE].

[33] M.R. Gaberdiel, A. Taormina, R. Volpato and K. Wendland, A K3 sigma model with $\mathbb{Z}_{2}^{8}$ : $\mathbb{M}_{20}$ symmetry, JHEP 02 (2014) 022 [arXiv:1309.4127] [INSPIRE].

[34] A. Taormina and K. Wendland, The symmetries of the tetrahedral Kummer surface in the Mathieu group $M_{24}$, arXiv:1008.0954 [INSPIRE].

[35] M.R. Gaberdiel, C.A. Keller and H. Paul, Mathieu Moonshine and symmetry surfing, J. Phys. A 50 (2017) 474002 [arXiv: 1609.09302] [INSPIRE].

[36] K. Wendland, Snapshots of conformal field theory, in the proceedings of the Winter School in Mathematical Physics: Mathematical Aspects of Quantum Field Theory, January

29-February 3, Les Houches, France (2015), arXiv:1404.3108 [INSPIRE].

[37] F. Malikov, V. Schechtman and A. Vaintrob, Chiral de Rham complex, Commun. Math. Phys. 204 (1999) 439 [math/9803041] [INSPIRE].

[38] L.A. Borisov, Vertex algebras and mirror symmetry, Commun. Math. Phys. 215 (2001) 517 [math/9809094] [INSPIRE].

[39] L. Borisov and A. Libgober, Elliptic genera of toric varieties and applications to mirror symmetry, Invent. Math. 140 (2000) 453 [math/9904126].

[40] V. Gorbounov and F. Malikov, Vertex algebras and the Landau-Ginzburg/Calabi-Yau correspondence, Mosc. Math. J. 4 (2004) 729 [math/0308114] [INSPIRE].

[41] B. Lian and A. Linshaw, Chiral equivariant cohomology. I., Adv. Math. 209 (2007) 99 [math/0501084].

[42] D. Ben-Zvi, R. Heluani and M. Szczesny, Supersymmetry of the chiral de Rham complex, Compos. Math. 144 (2008) 503 [math/0601532].

[43] A. Kapustin, Chiral de Rham complex and the half-twisted sigma-model, hep-th/0504074 [INSPIRE].

[44] M. Ademollo et al., Supersymmetric strings and color confinement, Phys. Lett. 62B (1976) 105 [INSPIRE]. 
[45] I. Frenkel and V. Kac, Basic representations of affine Lie algebras and dual resonance models, Invent. Math. 62 (1980/81) 23.

[46] G. Segal, Unitary representations of some infinite dimensional groups, Commun. Math. Phys. 80 (1981) 301 [INSPIRE].

[47] P. Goddard and D. Olive, Algebras, lattices and strings, in Vertex operators in mathematics and physics, I. Singer and J. Lepowsky ed., Math. Sci. Res. Inst. Publ. volume 3, Springer, Germany (1984), pp. 51-96.

[48] V. Kac, Vertex algebras for beginners, $2^{\text {nd }}$ edition, University Lecture Series volume 10, American Mathematical Society, Providence U.S.A. (1998). 